\title{
Brain glucose concentrations in poorly controlled diabetes mellitus as measured by high-field magnetic resonance spectroscopy
}

\author{
Elizabeth R. Seaquist ${ }^{\mathrm{a}, *}$, Ivan Tkac ${ }^{\mathrm{b}}$, Greg Damberg ${ }^{\mathrm{a}, 1}$, William Thomas ${ }^{\mathrm{c}}$, Rolf Gruetter ${ }^{\mathrm{b}, \mathrm{d}}$ \\ ${ }^{a}$ Department of Medicine, University of Minnesota Medical School, MN, 55455 USA \\ ${ }^{\mathrm{b}}$ Department of Radiology, University of Minnesota Medical School, MN, 55455 USA \\ ${ }^{\mathrm{c}}$ Division of Biostatistics, School of Public Health, University of Minnesota, MN, 55455 USA \\ ${ }^{\mathrm{d}}$ Department of Neuroscience, University of Minnesota Medical School, MN, 55455 USA \\ Received 23 September 2004; accepted 4 February 2005
}

\begin{abstract}
Hyperglycemia and diabetes alter the function and metabolism of many tissues. The effect on the brain remains poorly defined, but some animal data suggest that chronic hyperglycemia reduces rates of brain glucose transport and/or metabolism. To address this question in human beings, we measured glucose in the occipital cortex of patients with poorly controlled diabetes and healthy volunteers at the same levels of plasma glucose using proton magnetic resonance spectroscopy. Fourteen patients with poorly controlled diabetes (hemoglobin $\mathrm{A}_{1 \mathrm{c}}=9.8 \% \pm$ $1.7 \%$, mean \pm SD) and 14 healthy volunteers similar with respect to age, sex, and body mass index were studied at a plasma glucose of 300 $\mathrm{mg} / \mathrm{dL}$. Brain glucose concentrations of patients with poorly controlled diabetes were lower but not statistically different from those of control subjects $(4.7 \pm 0.9$ vs $5.3 \pm 1.1 \mu \mathrm{mol} / \mathrm{g}$ wet wt; $P=.1)$. Our sample size gave $80 \%$ power to detect a difference as small as $1.1 \mu \mathrm{mol} / \mathrm{g}$ wet wt. We conclude that chronic hyperglycemia in diabetes does not alter brain glucose concentrations in human subjects.

(C) 2005 Elsevier Inc. All rights reserved.
\end{abstract}

\section{Introduction}

The effects of chronic hyperglycemia on the brain have been difficult to define. Reduced cognitive function, particularly with respect to memory and attention, has been identified in patients with poorly controlled diabetes [1-3], and epidemiological studies demonstrate a link between dementia and a diagnosis of diabetes [4,5]. Investigators have identified specific abnormalities in central nerve conduction that are more common in diabetes [6-8], but it is unclear if these abnormalities are linked to metabolic changes that occur as a result of chronic hyperglycemia or are caused by the vascular disease so common in patients with diabetes.

Overall levels of glycemia have been proposed to play a role in determining brain glucose uptake and metabolism [9]. Because normal glucose sensing in the brain

* Corresponding author. MMC 101, MN 55455, USA. Tel.: +1 612 626 1960; fax: +1 6126263133 .

E-mail address: seaqu001@umn.edu (E.R. Seaquist).

${ }^{1}$ Current address: Park Nicollet Clinic. relies in part on rates of brain glucose uptake and metabolism, abnormalities in glucose sensing should be expected in subjects with aberrations in glucose homeostasis. Observations made in human subjects with diabetes suggest that these anticipated changes in glucose sensing do indeed occur. Subjects with recurrent hypoglycemia demonstrate a decrease in the blood glucose concentration at which they develop symptoms of hypoglycemia or mount a counterregulatory response to hypoglycemia [10-13]. Conversely, chronic hyperglycemia has been associated with an increase in the blood glucose concentration at which the symptoms of hypoglycemia (increased heart rate, release of counterregulatory hormones, etc) occur [14]. One plausible mechanism that has been proposed and investigated is that brain glucose uptake is changed in such a way as to compensate for the altered glucose delivery to the brain [9]. Glucose transport at the blood-brain barrier is predominantly mediated by the ubiquitous glucose transporter, GLUT1 [15-18]. In the setting of chronic hypoglycemia, glucose transporters at the blood-brain barrier have been found to be increased by some [19,20] but not all [21] investigators. After chronic hyperglycemia, glucose transporters 
at the rodent blood-brain barrier have been found to be reduced [19,22-24]. Together, these studies suggest that glucose transport may be one mechanism through which the brain can compensate for alterations in global glucose metabolism.

If altered expression of GLUT1 occurs as a result of aberrations in glucose homeostasis, brain glucose concentrations in subjects with such aberrations should be different from those in control subjects with chronic normoglycemia. Assuming a fixed glucose transport capacity on a per GLUT1 molecule basis, the decrease in transporter density that has been shown to occur in chronic hyperglycemia $[19,22-24]$ would be expected to lead to a decreased permeability surface area, which in turn would lead to a decreased apparent maximal glucose transport rate, $T_{\max }$. Without a major adaptive change in brain energy metabolism, which almost entirely relies on blood glucose and its transport across the blood-brain barrier, a decrease in $T_{\max }$ would be expected to lead to decreased brain glucose content at a given plasma glucose concentration, which would imply that glucose transport could become rate limiting for metabolism at higher plasma glucose concentrations. Regardless of the mechanism, it would appear that a change in brain glucose concentration at a given plasma glucose concentration is central to the hypothesis that brain glucose transport is a critical element underlying how the brain copes with changes in glucose availability.

Brain glucose concentrations can be directly measured using in vivo magnetic resonance spectroscopy (MRS) $[25,26]$, whereas positron emission tomography can provide precise measurements of the glucose phosphorylation in the brain and, to some extent, of the unidirectional glucose transport from the operational parameter, $\mathrm{k}_{1}$. Positron emission tomography studies thus far have not reported a reduction in glucose transport or an increase in glucose metabolism in patients with poorly controlled diabetes [27,28], possibly because small but clinically significant differences may have been beyond the precision of these important studies. However, we have recently demonstrated that patients with type 1 diabetes and hypoglycemia unawareness have steady-state brain glucose concentrations that are $13 \%$ higher than those in healthy volunteers studied under the same metabolic conditions using proton MRS [29]. Proton MRS may be able to identify the small differences in brain glucose concentrations that are expected to result from an alteration in glucose transport if the hypothesis that antecedent hyperglycemia in poorly controlled patients with diabetes reduces brain glucose transport is true. Therefore, the aim of the current study was to examine in human beings whether chronic hyperglycemia alters glucose content by measuring brain glucose concentrations in patients with poorly controlled diabetes and healthy volunteers at the same levels of plasma glucose using proton MRS.

\section{Methods}

\subsection{Study participants}

Subjects with diabetes were recruited for study participation from the Endocrine Clinic of the University of Minnesota. To be included in the study, subjects were required to be older than 18 years, have a hemoglobin $\mathrm{A}_{1 \mathrm{c}}$ $\left(\mathrm{HgbA}_{1 \mathrm{c}}\right)$ of greater than $8.5 \%$ within 3 months of enrollment (for healthy subjects, the range is from $4.5 \%$ to $6.0 \%$ ), have no recent episodes of hypoglycemia, and fulfill the requirements for a magnetic resonance study, which, in addition to the usual magnetic resonance exclusion criteria, precluded subjects weighing more than $300 \mathrm{lb}$. Control subjects were recruited from the University of Minnesota and FairviewUniversity Medical Center communities and were similar to the patients with respect to age, body mass index, and sex. The study protocol was conducted according to procedures approved by the institutional review board.

\subsection{Protocol}

All subjects with diabetes were asked to monitor their blood glucose values at home before each meal and at bedtime for the week before the study. Subjects with hypoglycemia (blood glucose, $<70 \mathrm{mg} / \mathrm{dL}$ with or without symptoms) within 24 hours before the study were excluded from participation. Patients with self-reported diagnosis of type 1 diabetes were admitted to the General Clinical Research Center of the University of Minnesota the night before the experiment. Their evening dose of long-acting insulin was held and they were maintained on an intravenous infusion of insulin overnight to maintain their glycemia between 100 and $180 \mathrm{mg} / \mathrm{dL}$. The insulin infusion was discontinued at 7:30 AM and the patients were then transported to the Center for Magnetic Resonance Research for the experiment. Subjects with a selfreported diagnosis of type 2 diabetes were asked to discontinue their oral medications 3 days before study and to hold their insulin the night before the experiment. Medications used alone or in combination by the 6 subjects with type 2 diabetes included troglitazone, insulin $(n=4)$, metformin $(n=2)$, glyburide $(n=3)$, pioglitazone $(n=2)$, and glipizide.

All subjects were studied in the Center for Magnetic Resonance in the morning after an overnight fast. In preparation for the experiment, an intravenous catheter was placed retrograde into one foot for the acquisition of blood samples and 2 additional intravenous catheters were placed into the upper extremities for the delivery of somatostatin, insulin, and glucose. The leg used for blood sampling was wrapped in heated towels and hot packs to arterialize the venous blood [30], and baseline samples were obtained for glucose, insulin, and ketones. A somatostatin infusion was then begun and advanced slowly to a rate of $0.16 \mathrm{mg} /(\mathrm{kg} \mathrm{min})$ over 30 minutes to suppress endogenous insulin secretion [31]. An intravenous insulin infusion 


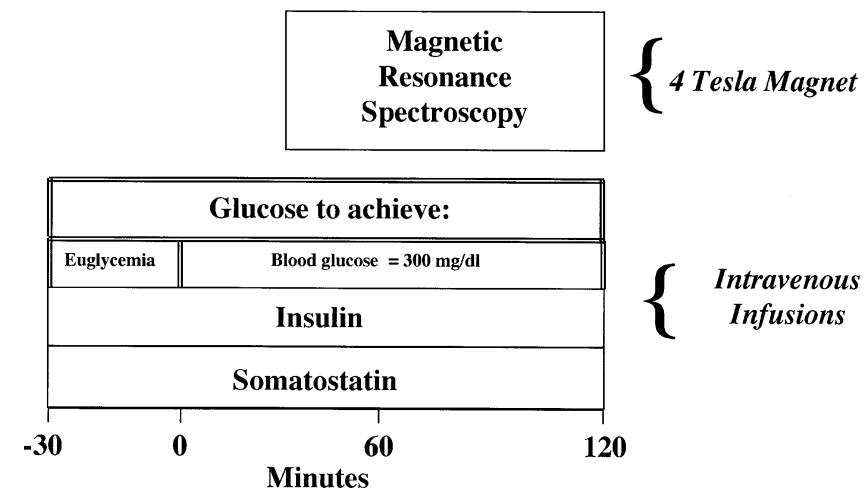

Fig. 1. Experimental protocol. Subjects were given intravenous infusions of somatostatin $[0.16 \mu \mathrm{g} /(\mathrm{kg} \mathrm{min})]$ and insulin $[0.5 \mathrm{mU} /(\mathrm{kg} \mathrm{min})]$ starting at time 0 . Glucose was then infused as necessary to maintain plasma glucose at $300 \mathrm{mg} / \mathrm{dL}$ between minutes 0 and 120. Data to measure cerebral glucose concentrations were acquired by MRS during the final 60 minutes of the study.

[0.5 mU/(kg min), Humulin Regular, Eli Lilly and Company, Indianapolis, Ind] was also begun at time 0. During the first 40 minutes, basal euglycemia was maintained by infusing glucose $(50 \%$ dextrose) at a variable rate. Thereafter, the glucose infusion was rapidly increased to clamp plasma concentration at $300 \mathrm{mg} / \mathrm{dL}$ (Fig. 1) a level we have previously shown to be high enough to yield a robust signal of the $\mathrm{H}-1$ proton of $\alpha$-glucose at $5.23 \mathrm{ppm}$, which was used for brain glucose quantification [32-34] (Fig. 2).

\subsection{Laboratory analyses}

Beginning with the start of the somatostatin and insulin infusions, blood samples were obtained every 5 minutes for determination of plasma glucose concentration in a nearby autoanalyzer (Beckman, Fullerton, Calif). Additional samples for subsequent determination of serum insulin concentrations by a Beckman Access instrument were obtained every 30 minutes. Hemoglobin $A_{1 c}$ was measured by highperformance liquid chromatography [35] in all subjects with diabetes.

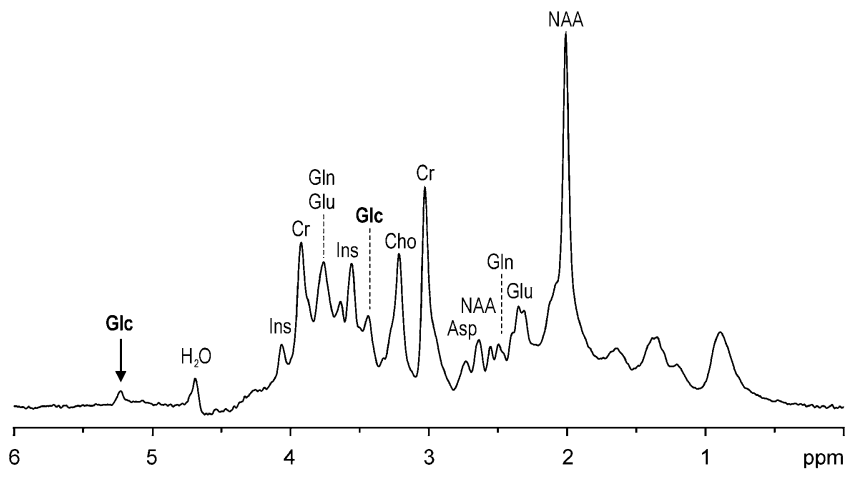

Fig. 2. In vivo ${ }^{1} \mathrm{H}$ magnetic resonance spectrum of a human brain during glucose infusion. In vivo ${ }^{1} \mathrm{H}$ MRS at $4 \mathrm{~T}$ was used to acquire a spectrum of a 15.6-mL volume of occipital cortex from a patient with diabetes when plasma glucose was clamped at $300 \mathrm{mg} / \mathrm{dL}$. The peak at $5.23 \mathrm{ppm}$ was used to quantify the brain glucose concentration as described in the text. Glc indicates glucose; Ins, inositol; Cho, choline; Glu, glutamate; NAA, $N$-acetylaspartate.

\subsection{Magnetic resonance spectroscopy}

Experiments were performed on a 4-T Siemens (Erlangen, Germany)/Oxford Magnet Technologies (Oxford, UK) magnet interfaced to a Varian console as detailed previously $[32,36]$. Subjects were placed supine on a bed above the surface coil and their heads were held in place by cushions. To minimize exposure to gradient noise, all subjects wore earplugs. A quadrature transmit/receive radio frequency (RF) coil consisting of 2 single-turn coils with a diameter of $14 \mathrm{~cm}$ was used [37]. A 16- to $27-\mathrm{mL}$ nominal volume of occipital cortex was selected for study. Localization of the signals was based on anatomical landmarks identified on T1-weighted modified driven equilibrium Fourier transform (MDEFT) [38] or rapid acquisition relaxation enhancement/ fast spin echo (RARE/FSE) imaging [39]. Localized shimming was performed using fast, automatic shim technique for mapping along projections (FASTMAP) $[40,41]$, which has resulted in consistent line shapes and line widths of the water resonance of 7 to $9 \mathrm{~Hz}$. For the measurements, we used $\mathrm{TE}=4-20$ milliseconds, $\mathrm{TM}=33$ milliseconds, and TR $=4.5$ milliseconds [42]. Water suppression was accomplished by the application of four 25-millisecond gaussian pulses [43] or by applying a series of RF pulses according to the variable power and optimized relaxation (VAPOR) scheme [44]. The water suppression did not affect signal by more than $5 \%$ outside $\pm 0.4 \mathrm{ppm}$ of the water signal. Outer volume saturation was achieved in slices adjacent to the volume of interest using hyperbolic secant pulses with variable RF power $[32,42,45]$. Data were saved in 10 blocks (16 scans per block) and then corrected for frequency drift (a few hertz) and summed. In each case, the plasma glucose concentrations were maintained at steady state for at least 20 minutes before spectral data were acquired. Data were acquired over a minimum of 20 minutes during which glycemia was clamped at target level.

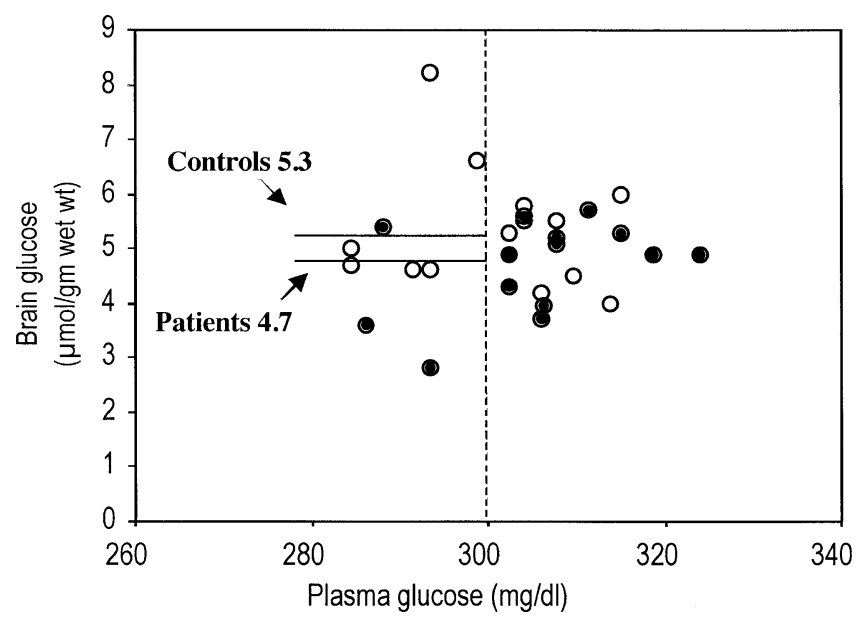

Fig. 3. Brain glucose concentrations. Fourteen subjects with diabetes and 14 matched control subjects were studied using the protocol described in the text. Mean glucose concentrations in brain and plasma for the diabetic participants (filled circles) and the healthy subjects (open circles) are shown. 
Table 1

Subject characteristics (mean \pm SD)

\begin{tabular}{llll}
\hline & Age $(\mathrm{y})$ & $\begin{array}{l}\text { Body mass } \\
\text { index }\left(\mathrm{kg} / \mathrm{m}^{2}\right)\end{array}$ & Sex $(\mathrm{M} / \mathrm{F})$ \\
\hline $\begin{array}{l}\text { Subjects with } \\
\text { diabetes }(\mathrm{n}=14)\end{array}$ & $43 \pm 13$ & $28.6 \pm 6.1$ & $5 / 9$ \\
$\begin{array}{l}\text { Healthy volunteers } \\
(\mathrm{n}=14)\end{array}$ & $37 \pm 9$ & $27.7 \pm 6.3$ & $5 / 9$ \\
$P$ & .161 & .702 & .999 \\
\hline
\end{tabular}

\subsection{Quantification of brain glucose}

Free induction decays were zero filled and apodized with $2 \mathrm{~Hz}$ of exponential line broadening. Peak areas of the glucose $(5.23 \mathrm{ppm})$, creatine $(\mathrm{Cr} ; 3.03 \mathrm{ppm})$, and macromolecule (2.98 ppm) resonances were quantified using peak fitting software supplied by the spectrometer manufacturer as described previously $[32,33]$. In brief, the area under the glucose peak at $5.23 \mathrm{ppm}$ was calculated relative to the area under the $\mathrm{Cr}$ methyl resonance at $3.04 \mathrm{ppm}$. The concentration of $\mathrm{Cr}$ was set to $10 \mathrm{mmol} / \mathrm{g}$ wet wt based on cortical concentrations of $9.6 \mathrm{mmol} / \mathrm{g}$ wet wt [46] and on contributions of $1 \mathrm{mmol} / \mathrm{g}$ wet wt $\gamma$-aminobutyric acid [47] and 1 to $2 \mathrm{mmol} / \mathrm{g}$ wet wt glutathione in this region of the brain $[48,49]$. Quantification of the glucose peak at $5.23 \mathrm{ppm}$ was performed and validated by comparison with measurements made using ${ }^{13} \mathrm{C}$-MRS [32].

\subsection{Statistical analysis}

Diabetic patients and control subjects were compared by 2 -sample Student $t$ test as appropriate.

\section{Results}

Fourteen subjects with diabetes and 14 healthy volunteers were studied. As shown in Table 1, the groups were similar with respect to age, body mass index, and sex. Eight of the patients had type 1 diabetes and 6 of the subjects had type 2 diabetes; their $\mathrm{HgbA}_{1 \mathrm{c}}$ was $9.8 \% \pm 1.7 \%$ on the day of the experiment. All subjects with diabetes except one with type 2 diabetes measured blood glucose concentrations at home as requested. The mean glucose value measured during the 4 days before the experiment was $203 \pm 27 \mathrm{mg} /$ dL. No correlation was found between the mean glucose values measured at home during the 4 days before the study and brain glucose concentration $(r=0.3 ; P=\mathrm{NS} ; \mathrm{n}=13)$. The relationship between glucose concentration in the brain and $\mathrm{HgbA}_{1 \mathrm{c}}$ also did not achieve statistical significance $(r=-0.076 ; P=\mathrm{NS} ; \mathrm{n}=14)$.

The plasma concentrations of glucose at the time of study were the same in both groups, which were accomplished using the glucose/insulin clamp technique (Table 2, Fig. 3). Serum insulin concentrations were significantly higher in the subjects with diabetes than in the control subjects $(P=.028$; Table 2, Fig. 3). Brain glucose concentrations in the subjects with diabetes were about $10 \%(0.6 \mu \mathrm{mol} / \mathrm{g})$ lower than in the control subjects but this difference was not statistically significant $(P=.10$; Table 2$)$. Adjusting the comparison for sex, age, or plasma glucose level gave the same finding. Our sample size gave $80 \%$ power to detect a difference in brain glucose concentration as small as $1.1 \mu \mathrm{mol} / \mathrm{g}$ wet wt.

\section{Discussion}

The present study found no significant difference between the brain glucose concentrations measured by MRS at $4 \mathrm{~T}$ in patients with poorly controlled diabetes and those in healthy volunteers studied at the same plasma glucose concentration. Although patients with poorly controlled diabetes had an average brain glucose concentration that was $0.6 \mu \mathrm{mol} / \mathrm{m}$ wet wt lower than in control subjects, our study only had $80 \%$ power to detect a difference from as small as $1.1 \mu \mathrm{mol} / \mathrm{g}$ wet wt. The clinical significance of any alteration smaller than 1.1 $\mu \mathrm{mol} / \mathrm{g}$ wet wt, particularly under conditions of euglycemia or hyperglycemia, is likely to be very small. It appears unlikely that poorly controlled diabetes has clinically meaningful effects on steady-state brain concentrations in human subjects.

Interestingly, recurrent hypoglycemia in patients with type 1 diabetes does appear to affect brain glucose transport and/or metabolism in human beings. We recently observed that brain glucose concentrations are significantly higher in patients with type 1 diabetes, recurrent and frequent hypoglycemia, and hypoglycemia unawareness than in control subjects studied under the same conditions [29], as would be expected if brain glucose transport increases and/or brain glucose metabolism decreases in response to hypoglycemia so as to ensure constant energy metabolism. Taken together, these findings may suggest that the brain is better able to compensate for conditions of glucose deficiency than for glucose excess. Alternatively, patients with type 1 diabetes and hypoglycemia unawareness may experience more prolonged or severe metabolic conditions that trigger the compensation in glucose transport and/or metabolism, as we

Table 2

Concentrations of glucose and insulin during the clamp study (mean \pm SD)

\begin{tabular}{llll}
\hline & Plasma glucose $(\mathrm{mg} / \mathrm{dL})$ & Serum insulin $(\mathrm{pmol} / \mathrm{L})$ & Brain glucose $(\mu \mathrm{mol} / \mathrm{g}$ wet wt) \\
\hline Subjects with diabetes & $306 \pm 11(\mathrm{n}=14)$ & $201 \pm 111(\mathrm{n}=14)$ & $4.7 \pm 0.9(\mathrm{n}=14)$ \\
Healthy volunteers & $301 \pm 11(\mathrm{n}=14)$ & $123 \pm 44(\mathrm{n}=10)$ & $5.3 \pm 1.1(\mathrm{n}=14)$ \\
$P$ & .253 & .028 & .100 \\
\hline
\end{tabular}


previously observed, than would the patients with poorly controlled diabetes included in this report. Although poorly controlled subjects with recent hypoglycemia were excluded from participation in the experiments reported, the magnitude of hyperglycemia experienced by these subjects in the weeks before the study was variable, as evidenced by the range of $\mathrm{HgbA}_{1 \mathrm{c}}$ from $7.9 \%$ to $13.2 \%$. The metabolic conditions in the previously studied patients with hypoglycemia unawareness were much more uniform in the weeks before the experiment, as demonstrated by their $\mathrm{HgbA}_{1 \mathrm{c}}$ of $6.9 \% \pm$ $0.2 \%$ (mean $\pm \mathrm{SEM}$ ).

In considering the data presented in this report, several factors that may have contributed to an underestimation of the true effect must be considered. First, plasma glucose concentrations in the patients were on average slightly higher by $5 \mathrm{mg} / \mathrm{dL}(P=.253)$ compared with those in the control subjects. Because several studies have reported a linear relationship between plasma and brain glucose concentrations [33,34,50], this difference would lead to an artifactual increase in the brain glucose concentration in the patients with diabetes. If this difference in plasma glucose between the groups is of any significance, the actual difference in brain glucose concentrations between the patients with diabetes and the control subjects should be larger than what we observed. However, with such a small and nonsignificant difference in blood glucose concentrations, it is unlikely that this would alter our results. A second factor that may have led to an underestimation of the effect of poorly controlled diabetes on brain glucose concentrations is that the subjects with diabetes had a significantly higher insulin concentration during the clamp study than did the control subjects. The increased plasma insulin concentration in the patient group may have been the result of subcutaneous depots of long-acting insulin or decreased insulin clearance in these subjects. We have recently demonstrated that insulin is without significant effect on the kinetics of cerebral glucose transport and/or metabolism in healthy subjects and that pharmacological concentrations of plasma insulin do not change brain glucose concentrations [34]. Interestingly, some studies have suggested effects of insulin on brain glucose transport and/or metabolism [51,52], but all these changes implicated that the hormone should have increased brain glucose content. Therefore, if insulin were to have an (as of yet undetected) effect on brain glucose content, it would have obscured any potential difference between poorly controlled patients and control subjects.

In summary, using MRS at $4 \mathrm{~T}$, we found no differences between the brain glucose concentrations in patients with poorly controlled diabetes and those in healthy subjects studied under similar levels of glycemia and insulinemia. Because we had sufficient power to detect a difference as small as $1.1 \mu \mathrm{mol} / \mathrm{g}$ wet $\mathrm{wt}$, it is unlikely that poorly controlled diabetes alters brain glucose transport and/or metabolism in a clinically significant manner.

\section{Acknowledgment}

This project was funded by grants from the National Institutes of Health, Bethesda, Md, RO1-NS35192 (ERS), MO1 RR00400, and P41 RR08079.

\section{References}

[1] Reaven GM, Thompson LW, Nahum D, Haskins E. Relationship between hyperglycemia and cognitive function in older NIDDM patients. Diabetes Care 1990;13:16-21.

[2] Perlmuter LC, Hakami MK, Hodgson-Harrington C, Ginsberg J, Katz $\mathrm{J}$, Singer DE, et al. Decreased cognitive function in aging non-insulindependent diabetic patients. Am J Med 1984;77:1043-8.

[3] Mooradian AD, Perryman K, Fitten J, Kavonian GD, Morley JE Cortical function in elderly non-insulin dependent diabetic patients. Behavioral and electrophysiologic studies. Arch Intern Med 1988;148: 2369-72.

[4] Leibson CL, Rocca WA, Hanson VA, Cha R, Kokmen E, O’Brien PC, et al. Risk of dementia among persons with diabetes mellitus: a population-based cohort study. Am J Epidemiol 1997;145:301 - 8.

[5] Arvanitakis Z, Wilson RS, Bienias JL, Evans DA, Bennett DA. Diabetes mellitus and risk of Alzheimer disease and decline in cognitive function. Arch Neurol 2004;61:661-6.

[6] Kurita A, Katayama K, Mochio S. Neurophysiological evidence for altered higher brain functions in NIDDM. Diabetes Care 1996;19:360 - 4

[7] Khardori R, Soler NG, Good DC, DevlescHoward AB, Broughton D, Walbert J. Brainstem auditory and visual evoked potentials in type 1 (insulin-dependent) diabetic patients. Diabetologia 1986;29:362-5.

[8] Dejgaard A, Gade A, Larsson H, Balle V, Parving A, Parving HH. Evidence for diabetic encephalopathy. Diabet Med 1991;8:162-7.

[9] Boyle PJ. Alteration in brain glucose metabolism induced by hypoglycaemia in man. Diabetologia 1997;40(Suppl 2):S69-74.

[10] Amiel SA, Sherwin RS, Simonson DC, Tamborlane WV. Effect of intensive insulin therapy on glycemic thresholds for counterregulatory hormone release. Diabetes 1988;37:901-7.

[11] Mitrakou A, Ryan C, Veneman T, Mokan M, Jenssen T, Kiss I, et al. Hierarchy of glycemic thresholds for counterregulatory hormone secretion, symptoms, and cerebral dysfunction. Am J Physiol 1991; 260:E67-74.

[12] Dagogo-Jack SE, Craft S, Cryer PE. Hypoglycemia-associated autonomic failure in insulin-dependent diabetes mellitus. Recent antecedent hypoglycemia reduces autonomic responses to, symptoms of, and defense against subsequent hypoglycemia. J Clin Invest 1993;91:819-28.

[13] Heller SR, Cryer PE. Reduced neuroendocrine and symptomatic responses to subsequent hypoglycemia after 1 episode of hypoglycemia in nondiabetic humans. Diabetes 1991;40:223-6.

[14] Boyle PJ, Schwartz NS, Shah SD, Clutter WE, Cryer PE. Plasma glucose concentrations at the onset of hypoglycemic symptoms in patients with poorly controlled diabetes and in nondiabetics. $\mathrm{N}$ Engl J Med 1988;318:1487-92.

[15] Lund-Andersen H. Transport of glucose from blood to brain. Physiol Rev 1979;59:305-52.

[16] Pardridge WM. Brain metabolism: a perspective from the blood-brain barrier. Physiol Rev 1983;63:1481 - 535

[17] Gjedde A. Blood-brain glucose transfer. In: Bradbury MWB, editor. Handbook of experimental pharmacology. New York: Springer Verlag; 1992. p. $67-117$.

[18] Pelligrino DA, LaManna JC, Duckrow RB, Bryan Jr RM, Harik SI. Hyperglycemia and blood-brain barrier glucose transport. J Cereb Blood Flow Metab 1992;12:887-99.

[19] Koranyi L, Bourey R, James D, Mueckler M, Fiedorek F, Permutt A. Glucose transporter gene expression in rat brain: pretranslational changes associated with chronic insulin-induced hypoglycemia, fasting, and diabetes. Mol Cell Neurosci 1991;2:244-52. 
[20] Kumagai AK, Kang YS, Boado RJ, Pardridge WM. Upregulation of blood-brain barrier GLUT1 glucose transporter protein and mRNA in experimental chronic hypoglycemia. Diabetes 1995;44:1399-404.

[21] Harik SI, LaManna JC. Vascular perfusion and blood-brain glucose transport in acute and chronic hyperglycemia. J Neurochem 1988;51: 1924-9.

[22] Pardridge WM, Triguero D, Farrell CR. Downregulation of bloodbrain barrier glucose transporter in experimental diabetes. Diabetes 1990;39:1040 - 4

[23] Lutz AJ, Pardridge WM. Insulin therapy normalizes GLUT1 glucose transporter mRNA but not immunoreactive transporter protein in streptozocin-diabetic rats. Metabolism 1993;42:939-44.

[24] Cornford EM, Hyman S, Cornford ME, Clare-Salzler M. Downregulation of blood-brain glucose transport in the hyperglycemic nonobese diabetic mouse. Neurochem Res 1995;20:869-73.

[25] Gruetter R, Novotny EJ, Boulware SD, Rothman DL, Mason GF, Shulman GI, et al. Direct measurement of brain glucose concentrations in humans by ${ }^{13} \mathrm{C}$ NMR spectroscopy. Proc Natl Acad Sci U S A 1992;89:1109-12.

[26] Gruetter R, Rothman DL, Novotny EJ, Shulman GI, Prichard JW, Shulman RG. Detection and assignment of the glucose signal in ${ }^{1} \mathrm{H}$ NMR difference spectra of the human brain. Magn Reson Med 1992;27:183-8.

[27] Fanelli CG, Dence CS, Markham J, Videen TO, Paramore DS, Cryer PE, et al. Blood-to-brain glucose transport and cerebral glucose metabolism are not reduced in poorly controlled type 1 diabetes. Diabetes 1998;47:1444-50.

[28] Brooks DJ, Gibbs JS, Sharp P, Herold S, Turton DR, Luthra SK, et al. Regional cerebral glucose transport in insulin-dependent diabetic patients studied using $\left[{ }^{11} \mathrm{C}\right] 3-O$-methyl-D-glucose and positron emission tomography. J Cereb Blood Flow Metab 1986;6:240 - 4.

[29] Criego A, Kumar A, Tran N, Tkac I, Gruetter R, Seaquist ER. Brain glucose concentrations in patients with type 1 diabetes and hypoglycemia unawareness. J Neurosci Res 2005;79:42-7.

[30] Seaquist ER. Comparison of arterialized venous sampling from the hand and foot in the assessment of in vivo glucose metabolism. Metabolism 1997;46:1364-6.

[31] Yen SS, Siler TM, DeVane GW. Effect of somatostatin in patients with acromegaly: suppression of growth hormone, prolactin, insulin and glucose levels. N Engl J Med 1974;290:935-8.

[32] Gruetter R, Garwood M, Ugurbil K, Seaquist ER. Observation of resolved glucose signals in ${ }^{1} \mathrm{H}$ NMR spectra of the human brain at 4 Tesla. Magn Reson Med 1996;36:1-6.

[33] Gruetter R, Ugurbil K, Seaquist ER. Steady-state cerebral glucose concentrations and transport in the human brain. J Neurochem 1998; 70:397-408.

[34] Seaquist ER, Damberg GS, Tkac I, Gruetter R. The effect of insulin on in vivo cerebral glucose concentrations and rates of glucose transport/ metabolism in humans. Diabetes 2001;50:2203-9.

[35] DCCT Research Group. Feasibility of centralized measurements of glycated hemoglobin in the Diabetes Control and Complications Trial: a multicenter study. Clin Chem 1987;33:2267-71.
[36] Seaquist ER, Gruetter R. Identification of a high concentration of scyllo-inositol in the brain of a healthy human subject using ${ }^{1} \mathrm{H}$ - and ${ }^{13}$ C-NMR. Magn Reson Med 1998;39:313 - 6.

[37] Adriany G, Gruetter R. A half-volume coil for efficient proton decoupling in humans at 4 Tesla. J Magn Reson 1997;125:178-84.

[38] Lee JH, Garwood M, Menon R, Adriany G, Andersen P, Truwit CL, et al. High contrast and fast three-dimensional magnetic resonance imaging at high fields. Magn Reson Med 1995;34:308-12.

[39] Hennig J, Nauerth A, Friedburg H. RARE imaging: a fast imaging method for clinical MR. Magn Reson Med 1986;3:823 - 33 .

[40] Gruetter R. Automatic, localized in vivo adjustment of all first- and second-order shim coils. Magn Reson Med 1993;29:804-11.

[41] Gruetter R, Tkac I. Field mapping without reference scan using asymmetric echo-planar techniques. Magn Reson Med 2000;43: 319-23.

[42] Tkac I, Seaquist ER, Gruetter RA. A comparison of several methods for quantification of resolved glucose signals in ${ }^{1}$ HNMR of the human brain. Proceedings of the Annual Meeting of the International Society of Magnetic Resonance in Medicine: 1938, 2000.

[43] Moonen CTW, van Zijl PCM. Highly effective water suppression for in vivo proton NMR spectroscopy (DRYSTREAM). J Magn Reson 1990;88:28-41.

[44] Tkac I, Starcuk Z, Choi IY, Gruetter R. In vivo ${ }^{1} \mathrm{H}$ NMR spectroscopy of rat brain at $1 \mathrm{~ms}$ echo time. Magn Reson Med 1999;41:649-56.

[45] de Graaf RA, Luo Y, Terpstra M, Garwood M. Spectral editing with adiabatic pulses. J Magn Reson B 1995;109:109-84.

[46] Petroff OA, Spencer DD, Alger JR, Prichard JW. High-field proton magnetic resonance spectroscopy of human cerebrum obtained during surgery for epilepsy. Neurology 1989;39:1197-202.

[47] Rothman DL, Petroff OA, Behar KL, Mattson RH. Localized ${ }^{1} \mathrm{H}$ NMR measurements of gamma-aminobutyric acid in human brain in vivo. Proc Natl Acad Sci U S A 1993;90:5662-6.

[48] Choi IY, Lei H, Gruetter R. Effect of deep pentobarbital anesthesia on neurotransmitter metabolism in vivo: on the correlation of total glucose consumption with glutamatergic action. J Cereb Blood Flow Metab 2002;22:1343-51.

[49] Terpstra M, Ugurbil K, Gruetter R. Direct in vivo measurement of human cerebral GABA concentration using MEGA-editing at 7 Tesla. Magn Reson Med 2002;47:1009-12.

[50] Choi IY, Lee SP, Kim SG, Gruetter R. In vivo measurements of brain glucose transport using the reversible Michaelis-Menten model and simultaneous measurements of cerebral blood flow changes during hypoglycemia. J Cereb Blood Flow Metab 2001;21:653-63.

[51] Namba H, Lucignani G, Nehlig A, Patlak C, Pettigrew K, Kennedy C, et al. Effects of insulin on hexose transport across blood-brain barrier in normoglycemia. Am J Physiol 1987;252:E299-303.

[52] Bingham EM, Hopkins D, Smith D, Pernet A, Hallett W, Reed L, et al. The role of insulin in human brain glucose metabolism: an ${ }^{18}$ fluorodeoxyglucose positron emission tomography study. Diabetes 2002;51: $3384-90$. 